\title{
Clinical and social factors associated with attention-deficit hyperactivity disorder medication use: population-based longitudinal study
}

\author{
Cédric Galéra, * Jean-Baptiste Pingault, * Grégory Michel, Manuel-Pierre Bouvard, Maria Melchior, \\ Bruno Falissard, Michel Boivin,** Richard E. Tremblay** and Sylvana M. Côté**
}

\section{Background}

The impact of longitudinal psychiatric comorbidity, parenting and social characteristics on attention-deficit hyperactivity disorder (ADHD) medication use is still poorly understood.

\section{Aims}

To assess the baseline and longitudinal influences of behavioural and environmental factors on ADHD medication use.

\section{Method}

Survival regressions with time-dependent covariates were used to model data from a population-based longitudinal birth cohort. The sample $(n=1920)$ was assessed from age 5 months to 10 years. Measures of children's psychiatric symptoms, parenting practices and social characteristics available at baseline and during follow-up were used to identify individual and family-level features associated with subsequent use of ADHD medication.

\section{Results}

Use of ADHD medication ranged from 0.2 to $8.6 \%$ between ages 3.5 to 10 years. Hyperactivity-inattention was the strongest predictor of medication use (hazard ratio $(\mathrm{HR})=2.75$, 95\% Cl 2.35-3.22). Among all social variables examined, low maternal education increased the likelihood of medication use $(\mathrm{HR}=2.09,95 \% \mathrm{Cl} 1.38-3.18)$ whereas immigrant status lowered this likelihood $(\mathrm{HR}=0.40,95 \% \mathrm{Cl}$ 0.17-0.92).

\section{Conclusions}

Beyond ADHD symptoms, the likelihood of receiving ADHD medication is predicted by social variables and not by psychiatric comorbidity or by parenting. This emphasises the need to improve global interventions by offering the same therapeutic opportunities (including medication) as those received by the rest of the population to some subgroups (i.e. immigrants) and by diminishing possible unnecessary prescriptions.

\section{Declaration of interest}

M.-P.B. has received financial support for the organisation of scientific meetings and was also the principal investigator in clinical trials for Shire and Lilly. In the past, C.G. received support from the industry to attend scientific congresses.
The first-line pharmacological intervention in attention-deficit hyperactivity disorder (ADHD) is stimulant medication, particularly methylphenidate. It has shown short-term efficacy in reducing behavioural symptoms of $\mathrm{ADHD}^{1,2}$ and improving cognitive tasks and academic performance., ${ }^{3,4}$ However, although use of medication for ADHD is associated with a possible decrease in criminality, ${ }^{5}$ there is little evidence of its impact on long-term impairments related to risky behaviours, psychiatric comorbidities and socio-occupational outcomes. ${ }^{6,7}$ Moreover, ADHD medication is generally well tolerated but potentially exposes children to adverse effects on appetite, growth, sleep and the cardiovascular system. ${ }^{8,9}$ In some cases ADHD medication is not adequately used since children without ADHD (false positives) or children with moderate forms can unduly be exposed to medication. Conversely, many children with severely impairing ADHD do not receive medication, even in countries with the highest rates of prescription. ${ }^{10}$ Accordingly, both expert assessment of diagnosis ${ }^{1,11,12}$ and expert monitoring and management of prescription practices are required. ${ }^{8}$ The international situation regarding ADHD medication prescription is variable. ${ }^{13}$ In many high-income countries, pharmacoepidemiological surveys suggest an increase in the pattern of stimulant medication use over time. ${ }^{14}$ This is notable in the USA, where the overall prevalence of medicated children rose from $0.6 \%$ in 1987 to $2.7 \%$ in 1997 and to $3.5 \%$ in 2008 , although

*These authors contributed equally to the work; **these authors share the senior authorship the situation varies from state to state (over- and underuse). ${ }^{15,16}$ However, in other countries, there is insufficient access to pharmacotherapy.

The substantial impact of ADHD on health and quality of life calls for a better understanding of its healthcare determinants, particularly medication. This is pressing, as alternative nonpharmacological interventions for ADHD (dietary and psychological treatments) have shown limited benefit for reducing core ADHD symptoms. ${ }^{17}$ Accordingly, medication still appears to be one of the most effective approaches to treating ADHD. However, there are risks of over-, under- and inadequate prescriptions. In addition, the concerns about the rising number of prescriptions, misuse and long-term safety have triggered worries among the stakeholders (the families of treated children, the media and the medical community), which may influence practices and acceptance of interventions. ${ }^{18,19}$ Identifying factors that predict medication use beyond ADHD symptoms is of utmost importance as this knowledge could be used by clinicians in their treatment decisions.

Prior research found that a variety of individual characteristics, sociodemographic and environmental factors are associated with the prescription of medication for ADHD: youth psychopathology (ADHD, externalising disorders, internalising disorders), being male, ethnicity, non-intact families, parental psychopathology, low maternal education, family income, negative family influences, low academic functioning, and previous receipt of stimulant medication. ${ }^{16,20-26}$ For some predictors, particularly disruptive comorbidity, divergent findings have been reported (protective 
in some studies $v$. risk factor in others). In addition, these studies had limitations: (a) medication use was often measured only at one point in time; (b) some studies were cross-sectional, implying a concomitant evaluation of risk factors and outcomes; (c) potential confounders were not taken into account; (d) a categorical diagnosis of psychiatric problems was generally used; (e) early childhood factors were not studied prospectively in a population-based sample. These limitations impeded the correct longitudinal appraisal of the role of risk factors, exposed the data to confounding biases and did not allow a dimensional approach to behavioural problems. The aim of the present study was to go beyond these limits by using a population-based birth cohort to assess the baseline and longitudinal influences of environmental and behavioural predictors on ADHD medication between the ages of 3.5 and 10 years. We tested the hypotheses that: (a) hyperactivity-impulsivity symptoms and inattention symptoms would predict medication use in this population-based sample; (b) other risk factors could heighten/lessen the likelihood of receiving ADHD medication.

\section{Method}

\section{Participants and procedure}

Data were drawn from the Quebec Longitudinal Study of Child Development (QLSCD). The QLSCD protocol was approved by the Quebec Institute of Statistics and the St Justine Hospital Research Center ethics committees. Data were collected by trained interviewers through repeated $(n=10)$ home interviews with the person most knowledgeable about the child (the mother for $98 \%$ of children) in order to obtain information on child, parent and family characteristics and behaviours. Informed written consent was obtained from all participating families at each assessment. Assessments were conducted at the following ages: 5 months, 1.5, 2.5, 3.5, 4, 5, 6, 7, 8 and 10 years. The initial sample was selected from birth registries and comprised 2120 children evaluated at 5 months and representative of children born in the province of Quebec (Canada) in 1997/1998. The average response rate over the 10 years of data collection was $83 \%$ (range: $63-100 \%$ ) with an average completeness of data equal to $79 \%$ (range: 61-91\%). The sample with complete data at the first assessment comprised 1920 children. This sample $(n=1920)$ was largely similar to the initial sample $(n=2120)$ regarding sociodemographic characteristics. However, there was a tendency for underprivileged families, non-intact families and immigrant families $(P<0.0001$ for all $)$ to have missing values. Table 1 describes the sociodemographic characteristics of the complete data sample at the first assessment $(n=1920)$.

\section{Measures}

\section{Outcome: use of ADHD medication}

Use of ADHD medication was reported by parents in a question referring to the preceding 12 months 'Does [your child] take any of the following prescribed medication on a regular basis: Ritalin or any other medication for treating hyperactivity or inattention?' at ages (years) 3.5 (0.2\% of the sample), $4(0.2 \%$ of the sample), $5(0.2 \%$ of the sample), $6(1.5 \%$ of the sample), $8(5.6 \%$ of the sample), and $10(8.6 \%$ of the sample).

\section{Explanatory variables}

Time-varying covariates: children's mental health and parenting. Symptoms of hyperactivity-impulsivity, inattention, anxiety, opposition and emotional problems were reported through the Interviewer Computerized Questionnaire when the children were $2.5,3.54,5,6$, and 8 years of age. Ratings relied on the early childhood behaviour scale from the Canadian National Longitudinal Study of Children and Youth. ${ }^{27}$ This tool incorporates items from the Child Behavior Checklist, ${ }^{28}$ the Ontario Child Health Study Scales ${ }^{29}$ and the Preschool Behavior Questionnaire. ${ }^{30}$ For each dimension the items used were as follows:

(a) hyperactivity-impulsivity: 'could not sit still, was restless or hyperactive', 'could not stop fidgeting,' 'was impulsive, acted without thinking', 'had difficulty waiting for his/her turn in games', 'couldn't settle down to do anything for more than a few moments';

(b) inattention: 'was unable to concentrate, could not pay attention for long', 'was easily distracted, had trouble sticking to any activity', 'was inattentive';

(c) anxiety: 'was too fearful or anxious', 'was worried', 'was nervous, high strung or tense', 'cried a lot';

(d) opposition: 'was defiant or refused to comply with adults' requests or rules', 'didn't seem to feel guilty after misbehaving', 'punishment didn't change his/her behavior', 'had temper tantrums or hot temper';

(e) emotional problems: 'seemed to be unhappy or sad', 'was not as happy as other children', 'had no energy, was feeling tired', 'had trouble enjoying him/herself'.

All items referred to the preceding 12 months and were coded on a frequency scale (never or not true: 0 ; sometimes or somewhat true: 1; often or very true: 2) and quantitative scores derived from scales were $\mathrm{z}$-standardised. Owing to high correlations and to avoid multi-collinearity, hyperactivity-impulsivity and inattention were combined into a single variable hyperactivity-inattention.

The Parental Cognition and Conduct Toward the Infant scale $^{31}$ was used to assess coercive parenting when the children were $2.5,3.5,4,5,6$, and 8 years of age, using the following items: 'I have been angry with my child when he/she was particularly fussy', 'when my child cries, he/she gets on my nerves', 'I have raised my voice with, or shouted at, my child when he/she was particularly fussy', 'I have spanked my child when he/she was particularly fussy', 'I have lost my temper when my child was particularly fussy', 'I have left my child alone in his/her bedroom when he/she was particularly fussy', 'I have shaken my child when he/she was particularly fussy'. All items were rated on an 11-point scale (higher score, more coercive parenting).

\section{Baseline covariates}

The gender of the child was coded 1 for boys and 0 for girls. Family structure was coded 1 if the family was non-intact (i.e. single-parent families; or families composed of a couple, married or common-law, living with at least one child not born to them) and 0 if the family was intact (i.e. the child lives with his/her two biological parents regardless of the type of conjugal relationship). Maternal education was coded 1 if low (no high-school diploma) and 0 if medium (high-school or post-secondary diploma) or high (university degree). Maternal age at birth of the target child was coded 1 if 21 years or younger (10.5\%) and 0 if older than 21 years. Household income was computed according to Statistics Canada's guidelines accounting for the family zone of residence, the number of people in the household and the family income in the past year. Income was coded 1 if insufficient and 0 if sufficient. Parental immigration status corresponded to nonimmigrant (mother and father born in Canada) $v$. immigrant (mother or father born outside Canada; $74 \%$ belonging to racial/ethnic minorities). Maternal and paternal depressive 
symptoms were assessed with the abbreviated version (12 items) of the Center for Epidemiological Studies Depression Scale (CES-D). ${ }^{32}$ Parents reported the frequency of depressive symptoms in the previous week. Each item was coded on a four-point scale. Informant total ratings were z-standardised. All baseline variables were assessed when the target child was 5 months of age.

\section{Statistical analyses}

Longitudinal risk factors (i.e. multiple measurements) were treated as time-varying covariates in all analyses. We first described the sample characteristics and the patterns of unadjusted associations between the main risk factors and ADHD medication use by computing the instantaneous hazard (equivalent to the proportion of the population using ADHD medication per unit time) on the basis of Kaplan-Meier survival functions. Second, we conducted bivariate and multivariate analyses using survival analysis with time-dependent covariates ${ }^{33}$ to determine associations between baseline/longitudinal risk factors and subsequent use of an ADHD medication. The method made it possible to estimate hazard ratios (HR) for the use of ADHD medication (endpoints at waves: 3.5, 4, 5, 6, 8, and 10 years of age) adjusting for the effects of other time-varying covariates (measured at waves: 2.5, 3.5, 4, 5, 6, and 8 years of age). To select predictors included in the multivariate models, we estimated bivariate associations between risk factors and the outcome (survival analysis). Variables with $P<0.05$ were entered into the multivariate models. Each participant was specified as a cluster of correlated observations, as medication status at any given time can depend on previous medication status. A robust variance was thus estimated to account for this pattern. In order to test the robustness of the findings, sensitivity analyses were performed using multiple imputation models (number of imputation, 100) under the missing-at-random (MAR) nonresponse mechanism. Finally, interactions between independent variables kept in the final model were tested. A $P<0.05$ was considered statistically significant.

\section{Results}

Table 1 summarises the characteristics of the sample at baseline and the number of person-years and events during follow-up. Figure 1 shows the instantaneous hazard rates of ADHD medication use: raw rates (Fig. 1(a)), by levels of hyperactivityinattention symptoms $(>+1$ s.d., +1 s.d. to median, $<$ median $)$ (Fig. 1(b)), by levels of maternal education (low/medium/high) (Fig. 1(c)), and by parental immigration status (immigrant $v$. non-immigrant) (Fig.1(d)).

Table 2 provides the results of proportional hazards regressions with time-dependent covariates. It shows the hazard ratios and 95\% confidence intervals for the use of ADHD medication. The fully-adjusted model (number of persons-years observed, 9165; number of events, 210) was significant (Wald $\chi^{2}=88.1$, d.f. $\left.=8, P<0.0001\right)$. All variables respected the hazard proportional assumptions (the contribution of each predictor was constant over time) except family income, which was not significantly associated with the outcome in multivariate models. To deal with this issue, we stratified on this variable, which still controls for the variable but does not provide an estimate of its contribution. Hyperactivity-inattention, male gender, low maternal education and immigration status were significantly associated with the use of ADHD medication. There was no statistically significant interaction. Additional analyses using multiple imputed data showed the same patterns of associations:

\begin{tabular}{|c|c|}
\hline Characteristics & \\
\hline Participants at study entry year, $n$ & 1920 \\
\hline Person-years at risk, $n$ & 11520 \\
\hline Person-years observed, $n$ & 9165 \\
\hline Events (ADHD medication exposure), $n$ & 210 \\
\hline $\begin{array}{l}\text { Gender of child, \% } \\
\text { Female } \\
\text { Male }\end{array}$ & $\begin{array}{l}49.6 \\
50.4\end{array}$ \\
\hline $\begin{array}{l}\text { Parental immigration status, \% } \\
\text { Non-immigrant } \\
\text { Immigrant }\end{array}$ & $\begin{array}{l}86.4 \\
13.6\end{array}$ \\
\hline $\begin{array}{l}\text { Maternal education, \% } \\
\text { No high-school diploma (low) } \\
\text { High-school/post-secondary diploma (medium) } \\
\text { University degree (high) }\end{array}$ & $\begin{array}{l}17.0 \\
55.3 \\
27.7\end{array}$ \\
\hline $\begin{array}{l}\text { Family status, \% } \\
\text { Intact } \\
\text { Non-intact }\end{array}$ & $\begin{array}{l}82.0 \\
18.0\end{array}$ \\
\hline $\begin{array}{l}\text { Family income, \% } \\
\text { Sufficient } \\
\text { Insufficient }\end{array}$ & $\begin{array}{l}78.1 \\
21.9\end{array}$ \\
\hline
\end{tabular}

hyperactivity-inattention $(P<0.0001)$, gender of the child $(P<0.0001)$, low maternal education $(P=0.006)$ and immigration status $(P=0.036)$ were significantly associated with the use of ADHD medication. Complementary analyses dichotomising hyperactivity-inattention into two dimensions hyperactivityimpulsivity and inattention showed that both dimensions were significantly associated with ADHD medication exposure (online Table DS1).

\section{Discussion}

Potential risks and possible misprescription have led some clinicians to highlight the need for caution in medicating ADHD. ${ }^{18}$ Our findings suggest that ADHD medication is significantly predicted by core ADHD symptoms but also over and above these symptoms by additional risk factors: being male, low maternal education and immigrant status.

\section{Comparison with previous findings and interpretation}

As expected, the strongest clinical predictors of ADHD medication use were symptoms of hyperactivity-inattention. This finding is reassuring since hyperactivity-inattention symptoms are the core behavioural symptoms of ADHD explicitly targeted by this medication. Interestingly, both dimensions (i.e. hyperactivity-impulsivity and inattention) were independently related to medication. Comorbid psychiatric symptoms assessed longitudinally (i.e. anxiety, emotional problems and opposition) were not independently associated with ADHD medication use when other risk factors were accounted for. In other words, these other symptoms (particularly symptoms of opposition that are often correlated with ADHD symptoms) did not induce medication. This means that clinicians did not medicate children who showed oppositional/anxious problems beyond what their ADHD status required. These results are particularly relevant in the context of public discussions regarding the ethics of medicating youths with psychotropic medications. 
(a)

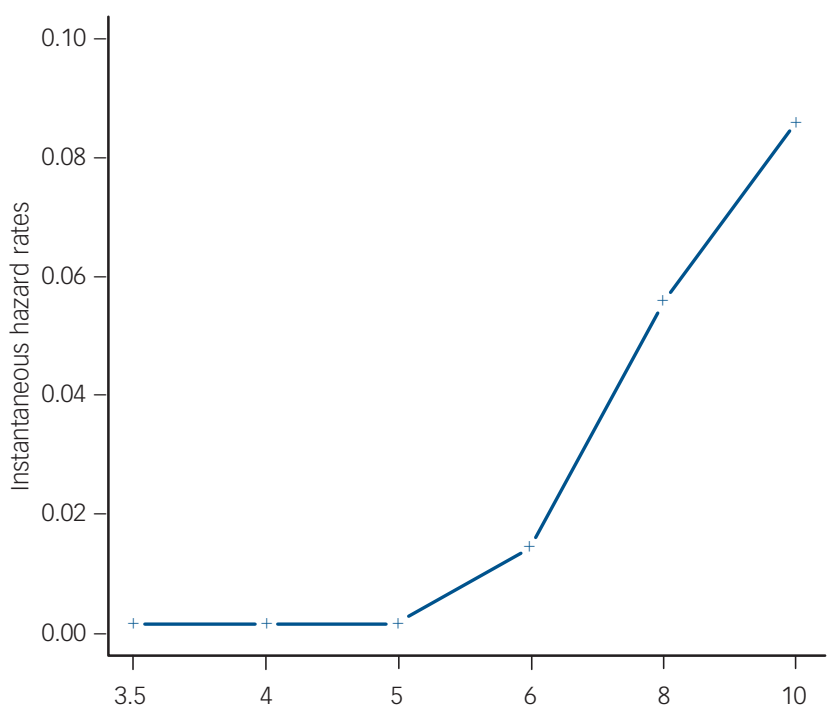

(c)

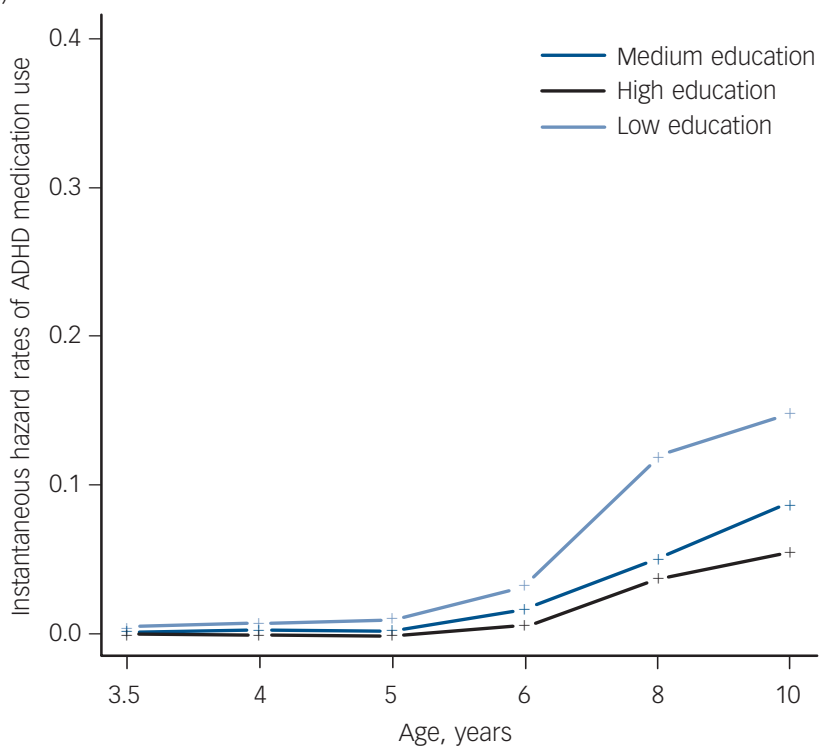

(b)

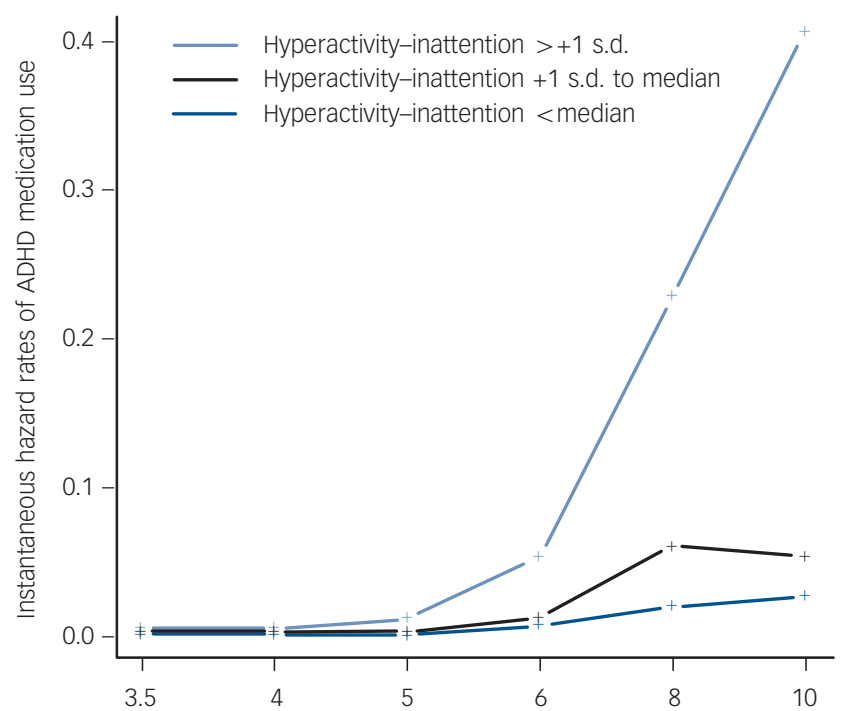

(d)

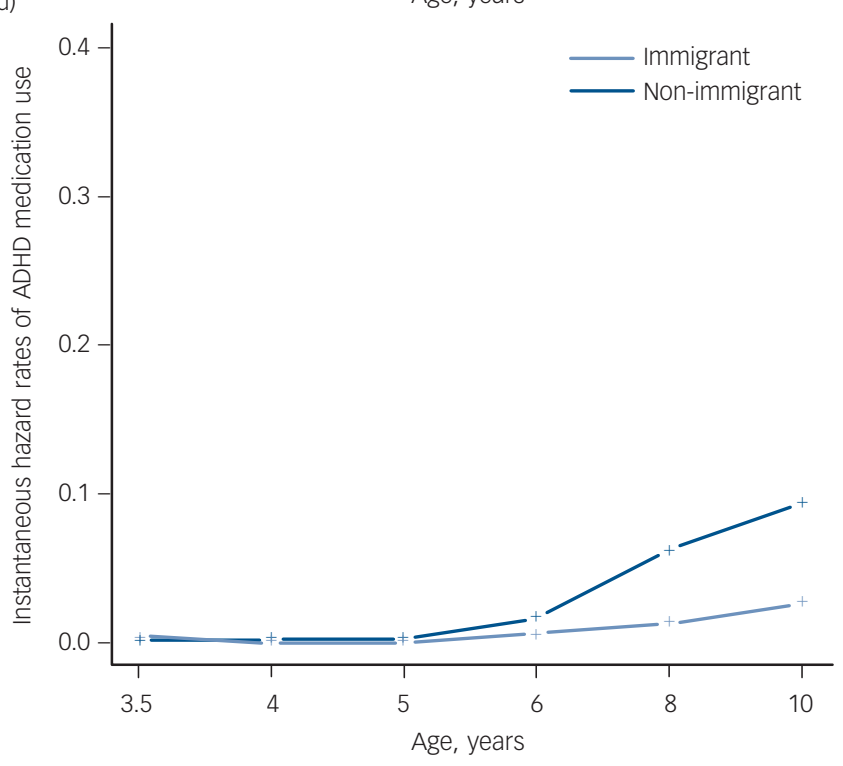

Fig. 1 Instantaneous hazards of attention-deficit hyperactivity disorder (ADHD) medication use.

(a) ADHD medication use; contribution of (b) hyperactivity-inattention; (c) maternal education; and (d) parental immigration status.

\section{Table 2 Survival models predicting exposure to attention-deficit hyperactivity disorder (ADHD) medication ${ }^{\mathrm{a}}$}

\begin{tabular}{|c|c|c|}
\hline & Unadjusted hazard ratio (95\% Cl) & Fully adjusted hazard ratio $(95 \% \mathrm{Cl})$ \\
\hline Male gender & $3.19(2.13-4.79)^{*}$ & $2.14(1.44-3.18)^{*}$ \\
\hline \multicolumn{3}{|l|}{ Psychiatric symptoms } \\
\hline Hyperactivity-inattention & $2.85(2.50-3.26)^{\star}$ & $2.75(2.35-3.22)^{\star}$ \\
\hline Anxiety & $1.29(1.09-1.52)^{\star}$ & $0.96(0.82-1.12)$ \\
\hline Opposition & $1.59(1.35-1.86)^{\star}$ & $0.85(0.71-1.01)$ \\
\hline Emotion & $1.09(0.94-1.27)$ & - \\
\hline Coercive parenting & $1.64(1.36-1.97)^{*}$ & $1.19(0.97-1.45)$ \\
\hline Immigrant & $0.28(0.12-0.66)^{*}$ & $0.40(0.17-0.92)^{*}$ \\
\hline Low maternal education & $2.47(1.65-3.69)^{*}$ & $2.09(1.38-3.18)^{*}$ \\
\hline Non-intact family & $1.89(1.24-2.88)^{*}$ & $1.36(0.86-2.15)$ \\
\hline Insufficient family income & $1.60(1.07-2.40)^{*}$ & - \\
\hline Young maternal age at birth & $1.56(0.95-2.57)$ & - \\
\hline Paternal depression & $1.08(0.92-1.28)$ & - \\
\hline Maternal depression & $1.08(0.90-1.31)$ & - \\
\hline $\begin{array}{l}\text { Data are courtesy of the Quebec Institute of Statistics. } \\
\star P<0.05 \text {. }\end{array}$ & & \\
\hline
\end{tabular}


Boys were more likely than girls to receive medication, even when controlling for the frequency of hyperactivity-inattention symptoms. This association could be related to a diagnostic bias towards boys. This could be attributable to a noisier clinical expression of the disorder in boys ${ }^{34}$ and/or to cultural trends (mothering ideology and masculinity stereotypes). ${ }^{18}$ Consequently, boys could be more likely to benefit from a more precise ADHD diagnosis and treatment than girls.

Recent research on ADHD suggests that the disorder is a complex and aetiologically heterogeneous condition caused by a combination of genetic, environmental and epigenetic contributions. $^{35,36}$ It has been argued that social and cultural characteristics may influence both diagnosis and prescription. ${ }^{18}$ The current study found associations consistent with this view. Most importantly and consistent with prior research, ${ }^{22,24}$ low maternal education was associated with ADHD prescription. There are several possible explanations for this finding. First, clinicians may conclude that psychoeducational interventions alone are less efficient than medication because of parental difficulties and limited resources. This would lead them to opt more easily for medication that has a quick and direct effect on ADHD symptoms and may make the parents more available for psychoeducative interventions. Second, mothers with more education may more readily seek and access information on medication, which makes them sensitive to drug issues and consequently less prone to accept use of medication. Third, low maternal education could confer risk for more severe symptomatology through a combination of environmental and biological factors. Fourth, phenomena such as parent-blame (more particularly mother-blame linked to the mothering ideology) and parental feelings of inadequacy in response to children's disruptive behaviour are thought to possibly bias ADHD diagnosis/treatment. ${ }^{18,37}$ All these potential social mechanisms need to be tested further by using qualitative and quantitative methods (for example randomised controlled trials) to examine the reasons for clinicians to suggest, and/or parents to accept, medication in families with different education levels, and examine the short- and long-term effectiveness of the different choices (for example to confirm or refute the benefit of more frequent prescriptions in lower-educated families). It is useful to note that other social variables were related to ADHD medication in univariate analyses (coercive parenting, non-intact family, insufficient family income) but were not associated with the study outcome in multivariate analyses, suggesting more distal influences or a less important role in medication use.

Parental immigrant status (which was strongly associated with racial/ethnic minorities) was related to lower ADHD medication use. This result is coherent with findings showing lower rates of ADHD diagnosis and less use of ADHD medication in children with a family immigrant background. ${ }^{38,39}$ The negative association could have several causes. First, immigrant status may be a barrier to proper access to healthcare. It has been suggested that minorities could be underdiagnosed owing to lesser healthcare utilisation because of poorer resources. ${ }^{40}$ Second, parents from ethnic minority groups have been shown to report fewer ADHD symptoms than White parents, independently of their health or socioeconomic characteristics. ${ }^{41}$ This could be related to culturally different beliefs regarding children's problematic behaviours and resistance to drug treatment. Third, USA children benefiting from Medicaid programmes, who come more often from racial/ethnic minorities, are less likely to benefit from interventions meeting quality of care standards and display higher unmet mental needs. ${ }^{42}$ Our results suggest that these hypotheses require further investigation in countries with different cultures and different healthcare systems.

\section{Strengths and limitations}

This study has several strengths, including the nature of the sample, the time period encompassed and the analytic approach. First, the large community-based sample made it possible to extend inferences beyond clinical populations. Second, the repeated measurement of ADHD and other behavioural symptoms prior to medication exposure increases reliability and strengthens causal inferences in comparison with prior crosssectional studies. Third, since methylphenidate initiation most commonly begins between ages 5 to 9 years, ${ }^{26}$ this study provides an opportunity to study the predictors of prescription during this crucial period.

The study also has limitations. First, the study relies on parental reports, which makes the data subject to informant bias. Such reports might partly reflect higher or lower parental tolerance to ADHD behaviours. Of note, we adjusted for factors potentially associated with tolerance such as oppositional behaviours or non-intact family. Second, a full-blown categorical ADHD diagnosis was not a requirement. However, this limitation was offset by the use of a dimensional approach, thus providing a better assessment of the phenotype heterogeneity that leads to medication use. ${ }^{36}$ Third, there were no data on functional impairment related to ADHD symptoms. Future investigations should take impairment severity into account, in order to explore factors associated with prescriptions in moderate and severe forms of ADHD, which could help in understanding the issue of potential overdiagnosis and overtreatment. ${ }^{43}$ Fourth, information relating to non-pharmacological ADHD interventions was not available. Fifth, we could not consider potential mechanisms explaining the observed associations, for example cultural influences such as school pressure, parental expectations, community representations, mothering ideology, masculinity stereotypes and healthcare system representations. These should be examined in future work with randomised controlled trials in order to enhance our understanding of medication determinants. Finally, it is to be acknowledged that the influences of social variables on prescription depend on the environmental, cultural and legal context. The findings cannot be directly generalised to other country settings, especially newly industrialised nations and countries with lower prescription rates. Further research could benefit from cross-cultural designs and comparisons between different countries.

\section{Implications}

The study's findings have theoretical and practical implications. Physicians should bear in mind the possible contribution of social characteristics on their practices. Individual features of children are not the only variables influencing drug exposure. The role of social context (parental educational level, immigration status) and parenting style needs to be fully considered in order to choose effective therapeutic strategies. Caution needs to be taken since clinical decisions grounded solely on risk might stigmatise families and children on a long-term basis. However, within the set of possible explanations, both legitimate (i.e. accessibility to pharmacological and non-pharmacological interventions) and more questionable (i.e. social pressure) may link social variables to medication. An effort to design specific parenting and psychoeducative interventions for families with low educational levels may encourage clinicians to consider alternatives to drug prescription for this target population. ${ }^{44}$ Clinicians may want to take parenting styles into account before prescribing and also develop specific psychoeducational interventions dedicated to this population. Furthermore, the present study suggests that some subgroups with less access to their healthcare system and 
medication could benefit from selective interventions to enhance their access to psychosocial services and consequently be offered the same opportunities as the rest of the population (i.e. have equal access to good mental healthcare, defined as appropriate and unbiased use of ADHD medications).

Major clinical practice guidelines ${ }^{1,11,12}$ recommend that the management of the ADHD clinical situation should rely on the joint use of psychoeducation, psychotherapy and pharmacotherapy. The current findings support the recommendations regarding the necessity of enhancing child and family therapeutic education and physicians' knowledge of prescription determinants. Future research will need to provide a better understanding of parental and practitioners' views to determine what drives decision-making on treatment choice.

Cédric Galéra, MD, PhD, University of Bordeaux, Department of Child and Adolescent Psychiatry, Charles Perrens Hospital and INSERM, The Bordeaux Schoo of Public Health (Institut de Santé Publique, d'Epidémiologie et de Développement), Centre INSERM U897, Epidemiology-Biostatistics, Bordeaux, France; Jean-Baptiste Pingault, PhD, Research Unit on Children's Psychosocial Maladjustment, University of Montreal and Sainte-Justine Hospital, Montreal, Canada and INSERM U669, Paris, France; Grégory Michel, PhD, University of Bordeaux, Psychology and Quality of Life, BordeauX, France; Manuel-Pierre Bouvard, MD, University of Bordeaux, Department of Child and Adolescent PSychiatry, Charles Perrens Hospital, Bordeaux, France; Maria Melchior, SCD, INSERM U1018, Centre for Research in Epid France, Maria Melchior, SCD, INSERM U1018, Centre for Research in Epidemiology and Population Health, Epidemiology of Occupational and Social Determinants of Health, Villejuif, France; Bruno Falissard, MD, PhD, INSERM U669, Paris, France; Michel Boivin, PhD, International Laboratory for Child and Adolescent Mental Health Department of Psychology, Laval University, Quebec City, Canada and Institute of Genetic, Neurobiological and Social Foundations of Child Development, Tomsk State University, Russian Federation; Richard E. Tremblay, PhD, Research Unit on Children's Psychosocial Maladjustment, University of Montreal and Sainte-Justine Hospital, Montreal, International Laboratory for Child and Adolescent Mental Health, University of Montreal, Montreal, Departments of Pediatrics, Psychiatry, and Psychology, University of Montreal, Montreal, Canada and School of Public Health, Physiotherapy, and Population Sciences, University College Dublin, Ireland and Institute of Genetic, Neurobiological and Social Foundations of Child Development, Tomsk State University, Russian Federation; Sylvana M. Côté, PhD, Research Unit on Children's Psychosocial Maladjustment, University of Montreal and Sainte-Justine Hospital, Montreal and International Laboratory for Child and Adolescent Mental Health, University of Montreal, Montreal, Canada and Institute of Genetic, Neurobiological and Social Foundations of Child Development, Tomsk State University, Russian Federation

Correspondence: Dr Cédric Galéra, Department of Child and Adolescent Psychiatry, University of Bordeaux, CH Perrens, 121 rue de la Béchade, 33076 Bordeaux, France. Email: cedric.galera@u-bordeaux.fr

First received 15 Nov 2013, final revision 31 Mar 2014, accepted 16 May 2014

\section{Funding}

This study was funded by Quebec's Department of Health and Social Services, the Canadian Institutes of Health Research, the Social Sciences and Humanities Research Council of Canada, the Quebec Fund for Research on Society and Culture, the Quebe Fund for Research on Nature and Technology, the Health Research Fund of Quebec, Quebec's Ministry of Research, Science, and Technology, Human Resources Development Canada, Health Canada, the Lucie and André Chagnon Foundation, the National Science Foundation, the University of Montreal, Laval University and McGill University.

\section{Acknowledgements}

R.E.T. is the founding scientific director of the Quebec Longitudinal Study of Children. We thank the children and families whose ongoing participation made this study possible. We also acknowledge the considerable contribution of the staff and the coordinators of the Quebec Longitudinal Study of Child Development at the Quebec Institute of Statistics and the tireless work of all the interviewers who assessed the mothers and children during this study. We thank the staff of the Research Unit on Children's Psychosocial Maladjustmen for their help in data access, analyses and management.

\section{References}

1 Banaschewski T, Coghill D, Santosh P, Zuddas A, Asherson P, Buitelaar J et al. Long-acting medications for the hyperkinetic disorders. A systematic review and European treatment guideline. Eur Child Adolesc Psychiatry 2006; 15: 476-95.

2 Greenhill L, Kollins S, Abikoff $\mathrm{H}$, McCracken J, Riddle M, Swanson J, et al. Efficacy and safety of immediate-release methylphenidate treatment for preschoolers with ADHD. J Am Acad Child Adolesc Psychiatry 2006; 45: 1284-93.

3 Prasad V, Brogan E, Mulvaney C, Grainge M, Stanton W, Sayal K. How effective are drug treatments for children with ADHD at improving on-task behaviour and academic achievement in the school classroom? A systematic review and meta-analysis. Eur Child Adolesc Psychiatry 2013; 22: 203-16.

4 Loe IM, Feldman HM. Academic and educational outcomes of children with ADHD. J Pediatr Psychol 2007; 32: 643-54.

5 Lichtenstein $\mathrm{P}$, Larsson $\mathrm{H}$. Medication for attention deficit-hyperactivity disorder and criminality. N Engl J Med 2013; 368: 776.

6 van de Loo-Neus GH, Rommelse N, Buitelaar JK. To stop or not to stop? How long should medication treatment of attention-deficit hyperactivity disorder be extended? Eur Neuropsychopharmacol 2011; 21: 584-99.

7 Molina BS, Hinshaw SP, Eugene Arnold L, Swanson JM, Pelham WE, Hechtman $\mathrm{L}$, et al. Adolescent substance use in the multimodal treatment study of attention-deficit/hyperactivity disorder (ADHD) (MTA) as a function of childhood ADHD, random assignment to childhood treatments, and subsequent medication. J Am Acad Child Adolesc Psychiatry 2013; 52 250-63.

8 Cortese S, Holtmann M, Banaschewski T, Buitelaar J, Coghill D, Danckaerts $\mathrm{M}$, et al. European ADHD Guidelines Group. Practitioner review: current best practice in the management of adverse events during treatment with ADHD medications in children and adolescents. J Child Psychol Psychiatry 2013; 54: 227-46.

9 Food and Drug Administration. FDA Drug Safety Communication: Safety Review Update of Medications used to treat Attention-Deficit/Hyperactivity Disorder (ADHD) in Children and Young Adults. FDA, 2011 (http:// www.fda.gov/drugs/drugsafety/ucm277770.html).

10 Rey JM, Sawyer MG. Are psychostimulant drugs being used appropriately to treat child and adolescent disorders? Br J Psychiatry 2003; 182: 284-6.

11 National Institute for Health and Care Excellence. Attention Deficit Hyperactivity Disorder. Diagnosis and Management of ADHD in Children, Young People and Adults. NICE clinical guideline 72. NICE, 2008.

12 Subcommittee on Attention-Deficit/Hyperactivity Disorder; Steering Committee on Quality Improvement and Management, Wolraich M, Brown L, Brown RT, DuPaul G, Earls M, et al. ADHD: clinical practice guideline for the diagnosis, evaluation, and treatment of attention-deficit/hyperactivity disorder in children and adolescents. Pediatrics 2011; 128: 1007-22.

13 International Narcotics Control Board. Report of the International Narcotics Control Board for 2012. International Narcotics Control Board, 2012 (http:// www.incb.org/incb/fr/publications/annual-reports/annual-report-2012.html).

14 Scheffler RM, Hinshaw SP, Modrek S, Levine P. The global market for ADHD medications. Health Aff (Millwood) 2007; 26: 450-7.

15 Zuvekas SH, Vitiello B. Stimulant medication use in children: a 12-year perspective. Am J Psychiatry 2012; 169: 160-6.

16 Olfson M, Gameroff MJ, Marcus SC, Jensen PS. National trends in the treatment of attention deficit hyperactivity disorder. Am J Psychiatry 2003 160: 1071-7.

17 Sonuga-Barke EJ, Brandeis D, Cortese S, Daley D, Ferrin M, Holtmann M, et al. Nonpharmacological interventions for ADHD: systematic review and meta-analyses of randomized controlled trials of dietary and psychological treatments. Am J Psychiatry 2013; 170: 275-89.

18 Singh I. Beyond polemics: science and ethics of ADHD. Nat Rev Neurosci 2008; 9: 957-64

19 Graham J, Coghill D. Adverse effects of pharmacotherapies for attentiondeficit hyperactivity disorder: epidemiology, prevention and management CNS Drugs 2008; 22: 213-37.

20 Leslie LK, Wolraich ML. ADHD service use patterns in youth. J Pediatr Psychol 2007; 32: 695-710.

21 Bird HR, Shrout PE, Duarte CS, Shen S, Bauermeister JJ, Canino G. Longitudinal mental health service and medication use for ADHD among Puerto Rican youth in two contexts. I Am Acad Child Adolesc Psychiatry 2008; 47: 879-89.

22 Hjern A, Weitoft GR, Lindblad F. Social adversity predicts ADHD-medication in school children-a national cohort study. Acta Paediatr 2010; 99: 920-4.

23 Lindblad F, Ringbäck Weitoft G, Hjern A. Maternal and paternal psychopathology increases risk of offspring ADHD equally. Epidemiol Psychiatr Sci 2011; 20: 367-72.

24 Miller AR, Kohen D, Johnston C. Child characteristics and receipt of stimulant medications: a population-based study. Ambul Pediatr 2008; 8: 175-81.

25 Romano E, Thornhill S, Lacourse E. An 8-year follow-up study of profiles and predictors of methylphenidate use in a nationwide sample of boys. J Pediatr 2009; 155: 721-27.

26 Winterstein AG, Gerhard T, Shuster J, Zito J, Johnson M, Liu H, et al. Utilization of pharmacologic treatment in youths with attention deficit/ 
hyperactivity disorder in Medicaid database. Ann Pharmacother 2008; 42: 24-31.

27 Statistics Canada. Overview of Survey Instruments for 1994-1995 Data Collection, Cycle 1. Statistics Canada, 1995.

28 Achenbach TM. Child Behavior Checklist. Department of Psychiatry, University of Vermont, 1991.

29 Boyle MH, Offord DR, Racine $Y$, Sanford M, Szatmari P, Fleming JE. Evaluation of the original Ontario Child Health Study scales. Can J Psychiatry 1993; 38 : 397-405.

30 Tremblay RE, Desmarais-Gervais L, Gagnon C, Charlebois P. The Preschool Behavior Questionnaire: stability of its factor structure between cultures, sexes, ages and socioeconomic classes. Int J Behav Dev 1987; 10: 467-84.

31 Boivin M, Pérusse D, Dionne G, Saysset V, Zoccolillo M, Tarabulsy GM, et al The genetic-environmental etiology of parents' perceptions and self-assessed behaviours toward their 5-month-old infants in a large twin and singleton sample. J Child Psychol Psychiatry 2005; 46: 612-30.

32 Radloff LS. The CESD-D scale: a self-report depression scale for research in the general population. Appl Psychol Meas 1977; 1: 385-401.

33 Therneau T (2013). A Package for Survival Analysis in S. R Package Version 2.37. (http://CRAN.R-project.org/package = survival).

34 Rucklidge JJ. Gender differences in attention-deficit/hyperactivity disorder. Psychiatr Clin North Am 2010; 33: 357-73.

35 Galéra C, Côté SM, Bouvard MP, Pingault JB, Melchior M, Michel G, et al. Early risk factors for hyperactivity-impulsivity and inattention trajectories from age 17 months to 8 years. Arch Gen Psychiatry 2011; 68: 1267-75.
36 Sonuga-Barke EJ, Halperin JM. Developmental phenotypes and causal pathways in attention deficit/hyperactivity disorder: potential targets for early intervention? J Child Psychol Psychiatry 2010; 51: 368-89.

37 Singh I. Doing their jobs: mothering with Ritalin in a culture of mother-blame. Soc Sci Med 2004; 59: 1193-205.

38 Singh GK, Yu SM, Kogan MD. Health, chronic conditions, and behavioral risk disparities among U.S. immigrant children and adolescents. Public Health Rep 2013; 128: 463-79.

39 Knopf $H$, Holling $H$, Huss M, Schlack R. Prevalence, determinants and spectrum of attention-deficit hyperactivity disorder (ADHD) medication of children and adolescents in Germany: results of the German Health Interview and Examination Survey (KiGGS). BMJ Open 2012; 2: e000477.

40 Visser SN, Lesesne CA, Perou R. National estimates and factors associated with medication treatment for childhood attention-deficit/hyperactivity disorder. Pediatrics 2007; 119 (suppl 1): S99-106.

41 Pastor PN, Reuben CA. Racial and ethnic differences in ADHD and LD in young school-age children: parental reports in the National Health Interview Survey. Public Health Rep 2005; 120: 383-92.

42 Zima BT, Bussing R, Tang L, Zhang L, Ettner S, Belin TR, et al. Quality of care for childhood attention-deficit/hyperactivity disorder in a managed care Medicaid program. J Am Acad Child Adolesc Psychiatry 2010; 49: 1225-37.

43 Thomas R, Mitchell GK, Batstra L. Attention-deficit/hyperactivity disorder: are we helping or harming? BMJ 2013; 347: f6172.

44 Boisjoli R, Vitaro F, Lacourse É, Barker ED, Tremblay RE. Impact and clinical significance of a preventive intervention for disruptive boys: a 15 year follow-up. Br J Psychiatry 2007; 191: 415-9.

\section{Postpartum psychosis}

\section{Roch Cantwell}

Mercurial and kaleidoscopic in its presentation, defying categorisation, postpartum psychosis is the will o' the wisp of mental illnesses. Affecting one in 500 women, its onset is early, abrupt and dramatic. Confusion, perplexity and mood lability are typical but little is certain in this most elusive condition. What is predictable is the risk of recurrence, as high as one in two following subsequent pregnancies. No other psychiatric malady can be predicted with such certainty, offering unique opportunities for prevention. We may even be close to the essence of this chimera through increasing evidence of its close link with bipolar disorder. 